TRANSACTIONS OF THE

AMERICAN MATHEMATICAL SOCIETY

Volume 349, Number 11, November 1997, Pages 4465-4479

S 0002-9947(97)01628-0

\title{
ONLY SINGLE TWISTS ON UNKNOTS CAN PRODUCE COMPOSITE KNOTS
}

\author{
CHUICHIRO HAYASHI AND KIMIHIKO MOTEGI
}

\begin{abstract}
Let $K$ be a knot in the 3 -sphere $S^{3}$, and $D$ a disc in $S^{3}$ meeting $K$ transversely more than once in the interior.

For non-triviality we assume that $|K \cap D| \geq 2$ over all isotopy of $K$.

Let $K_{n}\left(\subset S^{3}\right)$ be a knot obtained from $K$ by cutting and $n$-twisting along the disc $D$ (or equivalently, performing $1 / n$-Dehn surgery on $\partial D$ ).

Then we prove the following: (1) If $K$ is a trivial knot and $K_{n}$ is a composite knot, then $|n| \leq 1$; (2) if $K$ is a composite knot without locally knotted arc in $S^{3}-\partial D$ and $K_{n}$ is also a composite knot, then $|n| \leq 2$.

We exhibit some examples which demonstrate that both results are sharp.

Independently Chaim Goodman-Strauss has obtained similar results in a quite different method.
\end{abstract}

\section{INTRODUCTION}

Let $K$ be a knot in the 3 -sphere $S^{3}$ and $D$ an arbitrarily fixed disc intersecting $K$ transversely in its interior. Suppose that $|K \cap D| \geq 2$ over all isotopies of $K$ in $S^{3}-\partial D$. Performing $1 / n$-surgery along $C=\partial D$, we obtain a new knot $K_{n}$ in $S^{3}$ as the image of $K$. We call $K_{n}$ a twisted knot if the original knot $K$ is unknotted in $S^{3}$. In the case where $K$ intersects $D$ in just two points, then a twisted knot $K_{n}$ is always prime ([11], [14]), which is a generalization of "Unknotting number one knots are prime [10]". Mathieu asked in [5] whether twisted knots are always prime. Several families of twisted knots are known to be composite ([8], [12], [6]). All the examples are \pm 1 -twist.

In [7], it is shown that a twisted knot $K_{n}$ can be composite only for $|n| \leq 5$. Recently Teragaito [13] has shown that 5 can be replaced by 2 . The main result in this paper is

Theorem 1.1. If a twisted knot $K_{n}$ is composite, then $n= \pm 1$.

We will prove also the following generalization. We say that $K$ has no locally knotted arc in $S^{3}-C$ if there is no 3 -ball $B^{3}$ in $S^{3}-C$ such that $\left(B^{3}, B^{3} \cap K\right)$ is a knotted ball pair.

Received by the editors October 13, 1994 and, in revised form, August 30, 1995.

1991 Mathematics Subject Classification. Primary 57M25.

Key words and phrases. Knot, twisting, primeness, Scharlemann cycle.

The first author was supported in part by Fellowships of the Japan Society for the Promotion of Science for Japanese Junior Scientists.

The second author was supported in part by Grant-in-Aid for Encouragement of Young Scientists 06740083, The Ministry of Education, Science and Culture and Nihon University Research Grant B94-0025. 


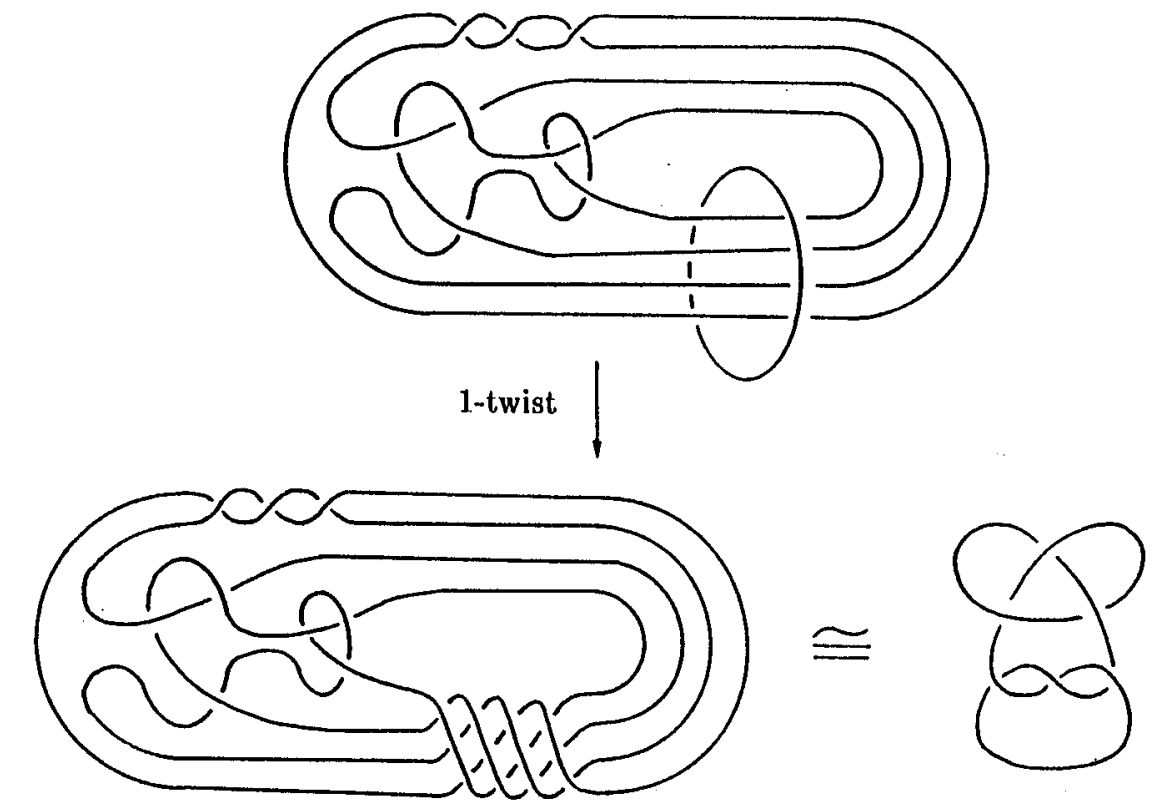

FigURE 1.1

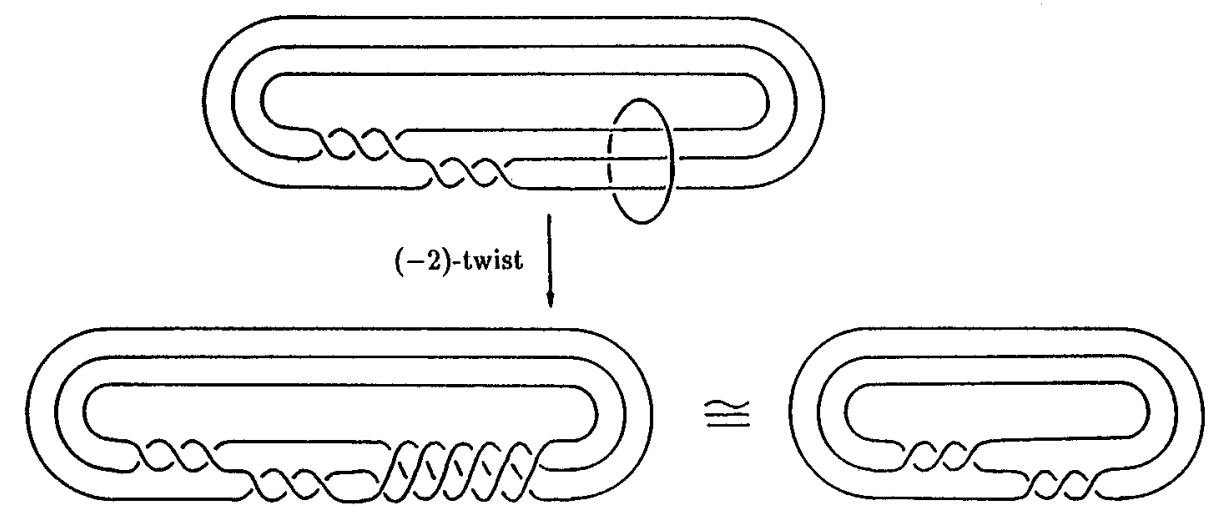

FiguRE 1.2

Theorem 1.2. Suppose that $K$ has no locally knotted arc in $S^{3}-C$. ( $K$ is possibly knotted in $S^{3}$.) If $K_{m}$ and $K_{n}$ are composite knots, then $|m-n| \leq 2$.

Theorem 1.2 is also sharp: there are examples such that $K_{m}$ and $K_{n}$ are both composite for $m, n$ with $|m-n|=1$ (Figure 1.1) and with $|m-n|=2$ (Figure 1.2). The first example is given by Tetsuo Shibuya. 
Independently, Chaim Goodman-Strauss has obtained Theorems 1.1 and 1.2 by a quite different method in his paper [2], in which he also shows that our example (Figure 1.2) is the only exceptional one for $|m-n|=2$.

\section{ACKNOWLEDGEMENTS}

We would like to thank Tetsuo Shibuya for sharing the example in Figure 1.1 with us. We are also grateful to Katura Miyazaki, Masakazu Teragaito and Akira Yasuhara for lots of discussions. This paper is inspired by [13]. Finally we would like to thank the referee and Peter B. Shalen for useful comments.

\section{INTERSECTION OF SURFACES AND ASSOCIATED GRAPHS}

To prove Theorems 1.1 and 1.2, it is sufficient to consider punctured annuli (derived from an essential annulus of composite knot exteriors) and punctured discs (derived from meridian discs of trivial knot exteriors).

However in this section we consider a more general situation as follows.

Let $M$ be an orientable 3 -manifold whose boundary $\partial M$ contains a toral component $T$.

Let $\left(F_{i}, \partial F_{i}\right) \subset(M, \partial M)(i=1,2)$ be an orientable surface, which is possibly compressible or $\partial$-compressible in $M$. Suppose that $\partial F_{i} \cap T \neq \emptyset$ and that the component of $\partial F_{1} \cap T$ and that of $\partial F_{2} \cap T$ have distinct slopes $\gamma_{1}$ and $\gamma_{2}$ on $T$. Then $\Delta=\Delta\left(\gamma_{1}, \gamma_{2}\right)$ will denote their minimal geometric intersection number. We assume that $F_{1}$ and $F_{2}$ intersect transversely and $\partial F_{1}$ and $\partial F_{2}$ intersect in the minimal number of points so that each component of $\partial F_{1}$ and that of $\partial F_{2}$ intersect just $\Delta$ times on $T$. We assign labels $1, \ldots, n_{i}$ to the components of $\partial F_{i} \cap T$ in the order which they appear on $T$. In the following we consider the case where $n_{i} \geq 2$ for $i=1,2$. Let $M\left(\gamma_{i}\right)$ denote the manifold obtained from $M$ by $\gamma_{i}$-Dehn filling along $T$, that is, by attaching a solid torus $V_{i}$ to $M$ along $T$ so that $\gamma_{i}$ bounds a disc in $V_{i}$. We obtain a surface $F\left(\gamma_{i}\right)$ when we cap off all the components of $\partial F_{i} \cap T$ with discs. We can regard the discs as meridian discs of $V_{i}$ and can suppose that $F\left(\gamma_{i}\right)$ is imbedded in $M\left(\gamma_{i}\right)$.

As in [3], we form the associated graph $G_{i}$ on $F\left(\gamma_{i}\right)$. Put $\{i, j\}=\{1,2\}$. Assign an arbitrary orientation to $F_{i}$ and we refer to + or - boundary components of $F_{i}$, according to the direction of the induced orientation of a boundary component as it lies on $T$. Label the end points of arc components of $F_{1} \cap F_{2}$ in $F_{i}$ with the corresponding labels of components of $\partial F_{j} \cap T$. Thus around each component of $\partial F_{i} \cap T$ we see the labels $\left\{1, \ldots, n_{j}\right\}$ appearing sequentially (either clockwise or anticlockwise according to the sign of the boundary) $\Delta$ times. Capping off all the boundary components of $\partial F_{i} \cap T$ with discs, we regard these discs as forming the "fat" vertices of the graph $G_{i}$ on the surface $F_{i}\left(\gamma_{i}\right)$. Every fat vertex $v$ has the sign according to the sign of the component of $\partial F_{i} \cap T$. The edges of $G_{i}$ are corresponding to the arcs of $F_{1} \cap F_{2}$ in $F_{i}$. Note that we neglect circle components of $F_{1} \cap F_{2}$. An edge $e$ is an interior edge if it connects vertices (possibly the same vertex), and a boundary edge otherwise. If $G_{1}$ has $k$ boundary edges, so does $G_{2}$, and vice versa. Let $e$ be an interior edge. Then we call $e$ a sign-preserving edge if it connects vertices which have the same sign, otherwise a sign-reversing edge. An edge is a loop if both its end points are in the same vertex. A loop is a sign-preserving edge. 


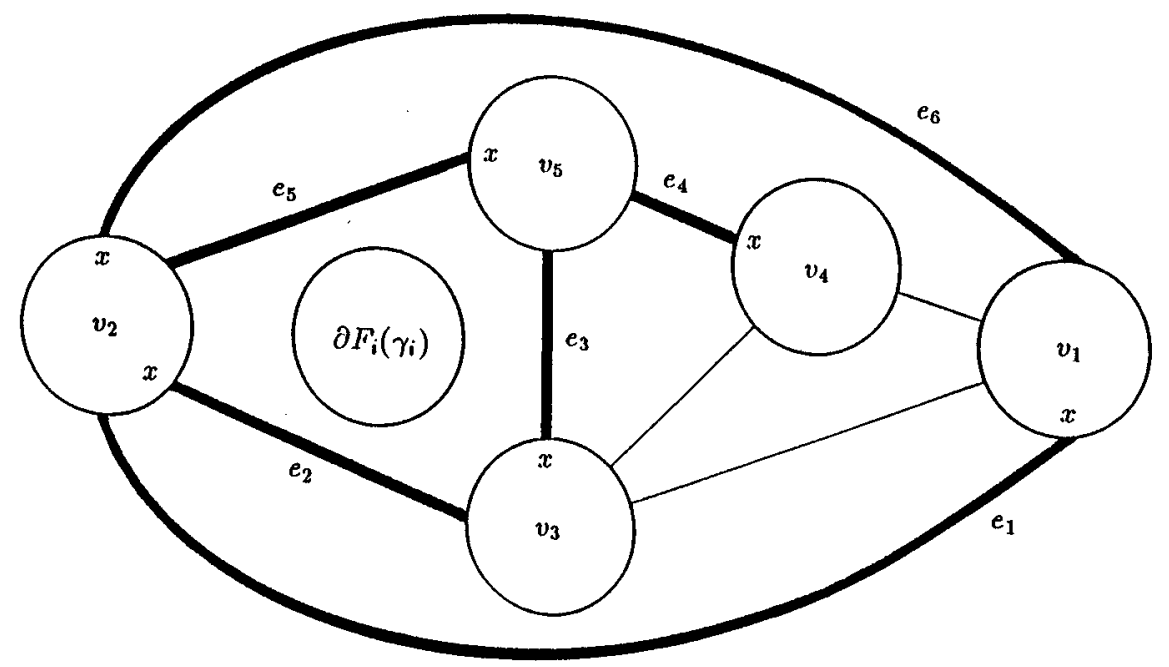

FiguRE 2.1

We thus obtain two labeled graphs, whose edges are in one to one correspondence. The orientability of $F_{i}(i=1,2)$ and $M$ gives us the parity rule - an edge $e$ in $G_{i}$ is a sign-preserving edge if and only if the corresponding edge in $G_{j}$ is a sign-reversing edge.

The edges and faces of $G_{i}$ are open edges and faces, i.e., components of $G_{i}-$ \{vertices\} and $F\left(\gamma_{i}\right)-G_{i}$, respectively. An edge $e$ is called a double edge of a face $P$ if $e \subset \operatorname{int} \bar{P}$, and a single edge otherwise. The boundary $\partial P$ is a "subgraph" which consists of vertices and edges intersecting $\bar{P}-P$. A face $P$ is called a disc face if $P$ is an open disc. Let $v$ be a fat vertex in $\partial P$ and $c$ a component of $\partial v-$ end point of edges in $\partial P\}$ which intersects $\bar{P}-P$. Then the closure of $c$ in $\partial v$ is called a corner of $\partial P$.

Let $x$ be a label of $G_{i}(i=1,2)$. An $x$-edge in $G_{i}$ is an edge with label $x$ at an end point.

A subgraph $\sigma$ is an $x$-edge cycle if $\sigma$ consists of sign-preserving $x$-edges and there is a disc face $E$ of the subgraph $\sigma$ such that $\sigma=\partial E$ (Figure 2.1). $E$ is called a face of the $x$-edge cycle.

The length of an $x$-edge cycle is defined to be (the number of single edges of the cycle $)+2 \times$ (the number of double edges of the cycle). For example, the cycle in Figure 2.1 has length 7. An $x$-edge cycle $\sigma$ is an $x$-cycle if $\sigma$ contains no double edges and if each edge of the cycle has the label $x$ at the head for some orientation of $\sigma$. If all the vertices in the face of an $x$-edge cycle (resp. $x$-cycle) have the same sign as those of the vertices of the cycle, then we call such an $x$-edge cycle (resp. $x$-cycle) a great $x$-edge cycle (resp. great $x$-cycle). A Scharlemann cycle is an $x$-cycle for some label $x$ which bounds a disc face of $G_{i}$. Note that if a Scharlemann cycle is an $x$-cycle, then we can regard it as an $(x+1)$-cycle or $(x-1)$-cycle by reversing the orientation. A Scharlemann cycle is called a trivial loop if its length equals one, and a non-trivial Scharlemann cycle otherwise. Let $t_{i}$ be the number of trivial loops in the graph $G_{i}$, and $b_{i}$ the number of boundary edges each of which 
has an end point in a fat vertex. Since $b_{1}=b_{2}$, we denote this number by $b$. Then we have the following inequality.

Theorem 2.1. If the graph $G_{i}$ does not contain non-trivial Scharlemann cycles for $i=1,2$, then we have

$$
\Delta\left(\gamma_{1}, \gamma_{2}\right) \leq 2+\left(t_{1}-\chi\left(F_{1}\left(\gamma_{1}\right)\right)\right) / n_{1}+\left(t_{2}-\chi\left(F_{2}\left(\gamma_{2}\right)\right)\right) / n_{2}+b / n_{1} n_{2} .
$$

Before proving this theorem, as applications of this, we will prove Theorems 1.1 and 1.2 in the following two sections.

The proof of Theorem 2.1 will be given in Sect. 6 .

\section{Proof of Theorem 1.1}

In this section we prove Theorem 1.1 by making use of Theorem 2.1 .

Let us assume that a twisted knot $K_{n}$ is composite. Put $M=S^{3}-\operatorname{int} N(K \cup C)$, $T=\partial N(C)$ and $T^{\prime}=\partial N(K)$. Slopes on $T, T^{\prime}$ will be parametrized by $\mathbb{Q} \cup\{1 / 0\}$ in the usual way, using a preferred meridian-longitude pair ([9]). Since $K \cup C$ forms an unsplittable link in $S^{3}, M$ is irreducible. Recall that $M(1 / 0)(\operatorname{resp} . M(1 / n)$ ) is a 3-manifold obtained from $M$ by attaching a solid torus $V_{1}=N(C)$ (resp. $\left.V_{2}\right)$ to $M$ along $T$ so that $\gamma_{1}=1 / 0$ (resp. $\left.\gamma_{2}=1 / n\right)$ bounds a disc in $V_{1}\left(\right.$ resp. $\left.V_{2}\right)$. The 3-manifold $M(1 / 0)$ is a solid torus, because the original knot $K$ is unknotted in $S^{3}$. We choose a meridian disc $D$ of the solid torus $M(1 / 0)=S^{3}-\operatorname{int} N(K)$ so that $D \cap N(C)$ is a disjoint union of meridian discs of $N(C)$ and $n_{1}=|D \cap N(C)|$ is minimal among all meridian discs of $M(1 / 0)$. Note that $n_{1} \geq 2$, otherwise $K_{n}$ would be also unknotted. Then $F_{1}=D \cap M$ is an incompressible and $\partial$-incompressible planar surface in $M$ because of the minimality of $n_{1}$. The surface $F_{1}$ has an outer boundary component in $T^{\prime}$ and $n_{1}$ inner boundary components each of which has the slope $\gamma_{1}=1 / 0$ on $T$.

Let $S$ be a decomposing 2 -sphere for the composite knot $K_{n}$. We can choose $S$ so that $S \cap V_{2}$ is a disjoint union of meridian discs of $V_{2}$ and $n_{2}=\left|S \cap V_{2}\right|$ is minimal. Note that $n_{2}$ is even since $S$ is separating in $S^{3}$. Because the trivial knot $K$ has no locally knotted arc in $S^{3}-C, n_{2} \neq 0$. Hence $n_{2} \geq 2$. Then $F_{2}=S \cap M$ is an incompressible, $\partial$-incompressible planar surface in $M$ because of the incompressibility of the annulus $F_{2}\left(\gamma_{2}\right) \subset M(1 / n)$ and of the minimality of $n_{2} . \quad F_{2}$ has two outer boundary components lying in $T^{\prime}$ and $n_{2}$ inner boundary components lying in $T$. Each outer boundary component has the slope $1 / 0$ on $T^{\prime}$ and each inner boundary component has the slope $\gamma_{2}=1 / n$ on $T$. By an isotopy of $F_{2}$, we may assume that $F_{1}$ and $F_{2}$ intersect transversely, and each outer boundary component of $F_{2}$ intersects the outer boundary component of $F_{1}$ exactly once, and each inner boundary component of $F_{2}$ intersects each inner boundary component of $F_{1}$ in $|n|$ points. By an innermost disc argument, we can assume that every circle component of $F_{1} \cap F_{2}$ bounds a disc neither in $F_{1}$ nor in $F_{2}$, since $F_{1}$ and $F_{2}$ are incompressible in $M$.

As in Sect. 2, we form associated graphs $G_{1}$ and $G_{2}$ on the surfaces $F_{1}\left(\gamma_{1}\right)$ and $F_{2}\left(\gamma_{2}\right)$ respectively. They have at most two boundary edges. Note that neither $G_{1}$ nor $G_{2}$ contains a trivial loop by the $\partial$-incompressibility of $F_{1}$ and $F_{2}$ and the minimality of the number of intersection points of $\partial F_{1}$ and $\partial F_{2}$ on $T$.

Sublemma 3.1 ([1, Lemma 2.5.2], [11, Proposition 5.6]). The graph $G_{i}$ cannot contain a non-trivial Scharlemann cycle for $i=1,2$. 
Proof. Suppose that $G_{1}$ contains a non-trivial Scharlemann cycle $\sigma_{1}$ which is simultaneously an $x$-cycle and an $(x+1)$-cycle. Let $P_{1}$ be the face of $\sigma_{1}$. The disc $\overline{P_{1}}$ is imbedded in the 3 -sphere $S_{2}^{3}=M(1 / n) \cup N\left(K_{n}\right)$. There is a 1-handle $H_{2}\left(\subset V_{2}\right)$ imbedded in $S_{2}^{3}$ such that $H_{2} \cap S=$ (the attaching discs $Q_{1}$ and $Q_{2}$ of $H_{2}$ ) and that $Q_{1}$ and $Q_{2}$ corresponding to the fat vertices of $G_{2}$ numbered $x$ and $(x+1)$. Let $S^{\prime}$ be the torus obtained by applying a surgery on $S$ along $H_{2}$. The boundary loop $\partial \overline{P_{1}}$ is imbedded in $S^{\prime}$, and the open disc of $P_{1}$ does not intersect $S \cup H_{2}$. Since $\sigma_{1}$ is a non-trivial Scharlemann cycle, the algebraic intersection number of $\partial \overline{P_{1}}$ and the co-core of the 1-handle $H_{2}$ is greater than or equal to two. Let $B^{3}$ be the 3-ball bounded by $S$ such that $\operatorname{int} B^{3} \cap H_{2}=\emptyset$. Then $B^{3} \cup H_{2} \cup N\left(\overline{P_{1}}\right)$ is a punctured lens space imbedded in $S_{2}^{3}$, a contradiction.

Suppose that $G_{2}$ contains a non-trivial Scharlemann cycle $\sigma_{2}$ which is simultaneously a $y$-cycle and $(y+1)$-cycle. Let $P_{2}$ be the face of $\sigma_{2}$. The disc $\overline{P_{2}}$ is imbedded in the 3 -sphere $S^{3}=M(1 / 0) \cup N(K)$. There is a 1-handle $H_{1}\left(\subset V_{1}\right)$ imbedded in $S^{3}$ such that $H_{1} \cap D=$ (the attaching discs $R_{1}$ and $R_{2}$ of $H_{1}$ ) and that $R_{1}$ and $R_{2}$ corresponding to the fat vertices of $G_{1}$ numbered $y$ and $(y+1)$. Thus we see that $N(D) \cup H_{1} \cup N\left(\overline{P_{2}}\right)$ is a punctured lens space, a contradiction.

Thus we can apply Theorem 2.1 and we have

$$
|n| \leq 2+\left(t_{1}-\chi\left(F_{1}(1 / 0)\right)\right) / n_{1}+\left(t_{2}-\chi\left(F_{2}(1 / n)\right)\right) / n_{2}+b /\left(n_{1} n_{2}\right) .
$$

Since $t_{1}=t_{2}=0, b \leq 2, \chi\left(F_{1}(1 / 0)\right)=1$ and $\chi\left(F_{2}(1 / n)\right)=0$, it follows that $|n| \leq 2+\left(2-n_{2}\right) /\left(n_{1} n_{2}\right)$. Hence if $n_{2} \geq 4$, we have $|n| \leq 1$ as desired.

In case $n_{2}=2$, Teragaito has proved in [13, Theorem 2] that $|n| \leq 1$. However for convenience of the readers we give a proof here following [13] with a slight modification. We will use Lemma 6.2 which is proved in Sect. 6 in this paper.

Since $S \cap V_{2}$ consists of two meridian discs of $V_{2}$, the graph $G_{2}$ has exactly two vertices $X$ and $Y$ whose signs are opposite.

If $b<2$ then Sublemma 3.1 and Theorem 2.1 implies that $|n| \leq 1$.

Hence in the following we assume that $b=2$. Note that the two boundary edges have end points in distinct boundary components of the annulus $\partial F_{2}\left(\gamma_{2}\right)$. If the two boundary edges are incident to the same vertex $X$, say, then there is a loop $\sigma$ at $Y$ since $X$ and $Y$ have the same valency. Since the two boundary edges form an essential arc on the annulus $F_{2}\left(\gamma_{2}\right), \sigma$ bounds a disc on $F_{2}\left(\gamma_{2}\right)$ which does not contain the vertex $X$. Hence there would be a trivial loop. This is a contradiction.

Thus for each vertex there is a single boundary edge incident to it in $G_{2}$. If there are more than $n_{1}+t_{1}-\chi\left(F_{1}\left(\gamma_{1}\right)\right)=n_{1}-1$ sign-reversing edges in $G_{2}$, then Lemma 6.2 (see Sect. 6) implies that $G_{1}$ contains a non-trivial Scharlemann cycle. Hence we can assume that there are at least $\left(\left(\left(|n| \times n_{1}-1\right) \times 2\right) / 2\right)-\left(n_{1}-1\right)$ sign-preserving edges in $G_{2}$, which are loops. Suppose that $|n| \geq 2$. Then there are more than or equal to $n_{1} / 2$ loops at the vertex $X$, say. Each loop must be essential in the annulus $F_{2}\left(\gamma_{2}\right)$. Consider the edge $e$ incident to $X$ immediately to the right of the boundary edge. Then $e$ must be a loop, for otherwise there would be a loop based at $X$ which is inessential in $F_{2}\left(\gamma_{2}\right)$. Similarly, the edge $e$ has the other end point immediately to the left of the boundary edge, and all the loops incident to $X$ are parallel to $e$. Among the loops, if $n_{1}$ is even then there is an edge whose two end points have the same label, which contradicts the parity rule. If $n_{1}$ is odd then there are two edges which form a non-trivial Scharlemann cycle of length 2 , which contradicts Sublemma 3.1. 


\section{Proof of Theorem 1.2}

We prove Theorem 1.2 as an application of Theorem 2.1.

Let $M$ be the solid torus $S^{3}-\operatorname{int} N(C)$ and $T$ the boundary $\partial M=\partial N(C)$. As in the proof of Theorem 1.1, we parametrize slopes on $T$ by $\mathbb{Q} \cup\{1 / 0\}$ using preferred meridian-longitude pair. Recall that $M(1 / m)$ (resp. $M(1 / n))$ is a 3 -sphere obtained from $M$ by attaching a solid torus $V_{1}$ (resp. $V_{2}$ ) to $M$ along $T$ so that the slope $\gamma_{1}=1 / m\left(\right.$ resp. $\left.\gamma_{2}=1 / n\right)$ bounds a disc in $V_{1}\left(\right.$ resp. $\left.V_{2}\right)$.

Let $S_{1}$ (resp. $S_{2}$ ) be a decomposing 2-sphere for the composite knot $K_{m}$ (resp. $K_{n}$ ), then $S_{1} \cap N\left(K_{m}\right)$ (resp. $S_{2} \cap N\left(K_{n}\right)$ ) consists of two meridian discs of $N\left(K_{m}\right)$ (resp. $N\left(K_{n}\right)$ ). We choose $S_{i}$ so that $S_{i} \cap V_{i}$ is a disjoint union of meridian discs of $V_{i}$ and that $n_{i}=\left|S_{i} \cap V_{i}\right|$ is minimal. Note that $n_{i} \geq 2$, because $K_{m}$ and $K_{n}$ have no locally knotted arcs in $S^{3}-C$.

Put $X=M-\operatorname{int} N(K)=S^{3}-\operatorname{int} N(K \cup C)$ and $T^{\prime}=\partial N(K)$. Then $P_{i}=S_{i} \cap X$ is an incompressible, $\partial$-incompressible planar surface in $X$. We may assume that $P_{1}$ and $P_{2}$ intersect transversely, that $\partial P_{1} \cap \partial P_{2} \cap T^{\prime}=\emptyset$, and that each component of $\partial P_{1} \cap T$ intersects each component of $\partial P_{2} \cap T$ in $|m-n|$ points on $T$. By an innermost disc argument, we can assume that every circle component of $P_{1} \cap P_{2}$ bounds a disc neither in $P_{1}$ nor in $P_{2}$.

Let $F_{i}$ be the surface $S_{i} \cap M$. As in Sect. 2, we form associated graphs $G_{i}$ on $S_{i}=F_{i}\left(\gamma_{i}\right)$. The graphs $G_{1}$ and $G_{2}$ have no boundary edges. Furthermore since $P_{1}$ (resp. $P_{2}$ ) is $\partial$-incompressible in $X$ and since $\partial P_{1}$ and $\partial P_{2}$ intersect in the minimal number of points on $T$, the face of a trivial loop of $G_{1}$ (resp. $G_{2}$ ) contains at least one of the two discs $S_{1} \cap N\left(K_{m}\right)$ (resp. $S_{2} \cap N\left(K_{n}\right)$ ). Hence $G_{i}$ contains at most two trivial loops, i.e., $t_{i} \leq 2$.

Sublemma 4.1. The graph $G_{i}$ cannot contain a non-trivial Scharlemann cycle for $i=1,2$.

Proof. Note that $M(1 / m)$ and $M(1 / n)$ are 3 -spheres. Suppose that one of $G_{1}$ and $G_{2}$, say $G_{1}$, contains a non-trivial Scharlemann cycle $\sigma$ which is simultaneously an $x$-cycle and an $(x+1)$-cycle. Let $P$ be the face of $\sigma$. The disc $\bar{P}$ is imbedded in the 3 -sphere $M(1 / n)$. There is a 1-handle $H\left(\subset V_{2}\right)$ imbedded in $M(1 / n)$ such that $H \cap S_{2}=$ (the attaching discs $Q_{1}$ and $Q_{2}$ of $H$ ) and that $Q_{1}$ and $Q_{2}$ are corresponding to the fat vertices of $G_{2}$ numbered with $x$ and $(x+1)$. Let $S_{2}^{\prime}$ be the torus obtained by applying a surgery on $S_{2}$ along $H$. The boundary of $\bar{P}$ is imbedded in $S_{2}^{\prime}$. The open disc $P$ may intersect $S_{2}$ in circles and does not intersect $H$. Each circle does not separate the two discs $Q_{1}$ and $Q_{2}$ on $S_{2}$ since there is an arc on $\partial \bar{P}$ connecting the two discs. Hence there is an innermost circle which on $S_{2}$ bounds a disc whose interior does not intersect $\bar{P}$. Let $B_{2}^{3}$ be the 3 -ball bounded by the 2 -sphere $S_{2}$ such that $\operatorname{int} B_{2}^{3} \cap H=\emptyset$. Then suitable surgery on $\bar{P}$ yields a new disc $P^{\prime}$ such that $P^{\prime} \cap\left(B_{2}^{3} \cup H\right)=P^{\prime} \cap S_{2}^{\prime}=\partial P^{\prime}=\partial \bar{P}$. Since $\sigma$ is a non-trivial Scharlemann cycle, $B_{2}^{3} \cup H \cup N\left(P^{\prime}\right)$ is a punctured lens space in the 3 -sphere $M(1 / n)$, a contradiction.

Since $\chi\left(F_{1}(1 / m)\right)=2, \chi\left(F_{2}(1 / n)\right)=2, t_{1} \leq 2, t_{2} \leq 2$ and $b=0$, we have $|m-n| \leq 2$ by using Theorem 2.1. This completes the proof. 


\section{Finding SCharlemann CYCles}

We work in the situation described in Sect. 2. Let $G_{i}$ be a graph on $F_{i}\left(\gamma_{i}\right)$. Our purpose in this section is to give a new condition which implies existence of a Scharlemann cycle.

Proposition 5.1. A face of a great $x$-edge cycle $\sigma$ of $G_{i}$ contains a face of a Scharlemann cycle.

Remark. If the surface $F_{j}$ is separating in the ambient 3-manifold $M$, then this result has been obtained by Gordon-Luecke [4]. However their proof does not work when $F_{j}$ is non-separating in $M$.

Proof. Let $P$ be the face of $\sigma$, and $G_{P}$ the subgraph $\left(G_{i} \cap P\right) \cup \partial P$. We take the subgraph $\Lambda$ of $G_{P}$ such that $\{$ the vertices of $\Lambda\}=\left\{\right.$ the vertices of $\left.G_{P}\right\}$ and that $\{$ the edges of $\Lambda\}=\left\{\right.$ the $x$-edges of $\left.G_{P}\right\}$. Since for each vertex $v$ of $\Lambda$ there is an $x$-edge incident to $v$ in $\bar{P}$, there exists a disc face $E$ of $\Lambda$ in $P$. The boundary $\partial E$ is an $x$-edge cycle and no corners of $\partial E$ contain a label $x$ in its interior.

Let $G_{E}$ be the graph $\left(G_{P} \cap E\right) \cup \partial E$. Note that $E$ contains no vertices of $G_{P}$. If $E$ contains a loop of $G_{E}$, then $E$ contains a face of a trivial Scharlemann cycle. Hence we assume that $E$ contains no loops.

If $\partial E$ is not a "circle" when we regard fat vertices as points, then we cut the graph $G_{E}$ along double edges of $\partial E$ and at fat vertices to which more than two edges of $\partial E$ are incident so that $\partial E$ deformed into a "circle". For example we cut the graph in Figure 2.1 into the graph in Figure 5.1. Let $\widehat{G_{E}}$ be the resulting graph, and $\widehat{E}$ the disc obtained by cutting $E$ as above.

It is sufficient to find a Scharlemann cycle in $\widehat{G_{E}}$ such that its face is in $\widehat{E}$. We will show this by induction on the length $\ell$ of the $x$-edge cycle $\partial \widehat{E}$.

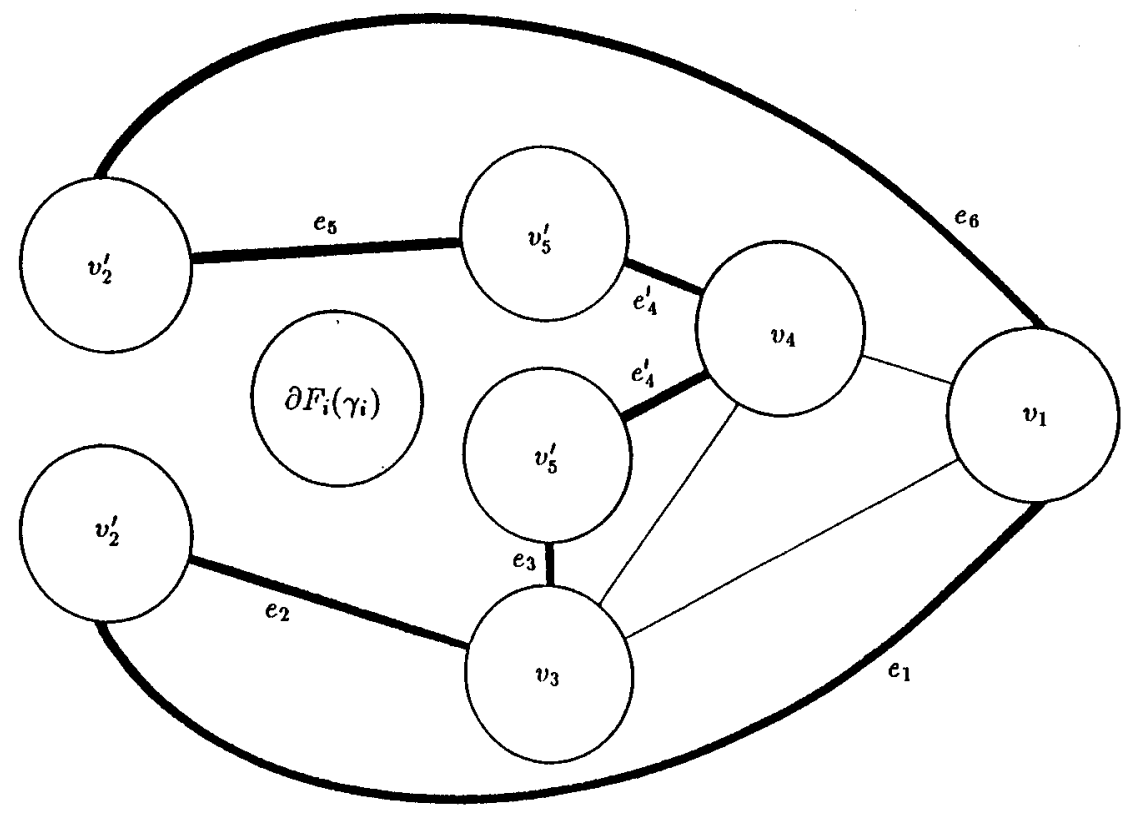

Figure 5.1 
To study the graph $\widehat{G_{E}}$, we forget the geometric meaning and consider a more general situation, which is purely abstract in the sense that the parity rule does not hold. More precisely we consider the following graphs.

Let $\Gamma$ be a graph on $F_{i}\left(\gamma_{i}\right)$ and $\sigma$ an $x$-edge cycle in $\Gamma$. Let $E$ be a disc face of $\sigma$. We assume that $\Gamma=(\Gamma \cap E) \cup \partial E$ (i.e., $\sigma$ is the outermost cycle of $\Gamma$ ). Further $\Gamma$ satisfies the following :

(1) $\sigma$ is homeomorphic to a circle when we regard fat vertices as points,

(2) $\Gamma$ has no vertices in $E$,

(3) each end of each edge is assigned a number $1,2, \ldots$, or $N(N \geq 2)$, and around each corner of $\sigma$ the ends are labelled $i, i+1, \ldots, i+k(\bmod N)$ for some $i$ and $k$ in, say, anticlockwise order, where integers $i$ and $k$ may vary from corner to corner,

(4) there is no loop in $E$.

Note that all edges in $\Gamma$ are sign-preserving edges and an $x$-edge cycle (resp. $x$-cycle) is automatically a great $x$-edge cycle (resp. great $x$-cycle) by the condition (2), and thus $\sigma$ is a great $x$-edge cycle.

We call the following condition the weak parity rule - every sign-preserving edge has distinct labels at end points. (Clearly the parity rule implies the weak parity rule. ) We call a sign-preserving edge which has the same label at both end points a squashed Scharlemann cycle, and continue to use the terminology established in Sect. 2 for the above graphs.

Lemma 5.2. If the weak parity rule holds for edges in the open disc $E$, then $\Gamma$ contains a non-squashed Scharlemann cycle (whose face is contained in E).

In our original situation, regarding $\widehat{G_{E}}$ as $\Gamma$ we find a Scharlemann cycle in $\widehat{G_{E}}$, hence in $G_{i}$, which establishes Proposition 5.1.

The remainder of this section is devoted to the proof of this lemma.

The proof will be done by induction on the length $\ell$ of $\sigma$. Considering the innermost $x$-edge cycle if necessary, we can assume that there are no $x$-edges in $E$.

Proof of Lemma 5.2. We start with the following sublemma.

Sublemma 5.3 (Special case of Lemma 5.2). Suppose that $\sigma=\partial E$ is an x-cycle. If the weak parity rule holds for edges in $E$, then $\Gamma$ contains a non-squashed Scharlemann cycle whose face is contained in $E$.

Proof. The proof of Lemma 2.6.2 [1] still works for this sublemma.

If $\partial E$ does not have a corner such that both its two end points (i.e., $\partial$ (corner)) have the label $x$ (we call such a corner an $x$ - $x$ corner), then $\partial E$ is an $x$-cycle. Hence by Sublemma 5.3, $E$ contains a non-squashed Scharlemann cycle.

We assume in the rest of the proof that there exists an $x$ - $x$ corner.

Sublemma 5.4. Assume that the weak parity rule holds for edges in E. If there is a family of more than $N / 2$ parallel edges in $\Gamma$, then we have:

(1) there is a non-squashed Scharlemann cycle (of length two) in that family and its face is contained in $E$, or

(2) $N$ is odd and the family consists of just $(N+1) / 2$ parallel edges including a squashed Scharlemann cycle which is an edge on $\partial E$. 
Proof. The sets of labels at the two ends of the parallel family are not disjoint. Hence there is a squashed Scharlemann cycle or an $x$-cycle (for some label $x$ ) in that family.

In the former case the squashed Scharlemann cycle is an edge of $\sigma$ and $N$ must be odd, otherwise $E$ contains a squashed Scharlemann cycle, a contradiction. If there are more than $(N+1) / 2$ parallel edges, then there is a non-squashed Scharlemann cycle of length two in that family.

In the latter case (i.e., there is an $x$-cycle), then Sublemma 5.3 implies existence of a non-squashed Scharlemann cycle.

First we consider the case $\ell=2$.

Since there is an $x-x$ corner, say $Q, \Gamma$ consists of $N+1$ parallel sign-preserving edges. Then Sublemma 5.4 shows that $\Gamma$ contains a non-squashed Scharlemann cycle.

Let us assume that $\ell \geq 3$.

An edge $e$ is called a diagonal edge if $e$ is incident to an $x-x$ corner $Q$ and has the other end point in a corner $R$ which is not adjacent to $Q$ on $\sigma$. When $\ell=3$, there is no diagonal edge.

We divide into two cases depending upon whether a diagonal edge $e$ exists or not.

Case 1: There is no diagonal edge on $\Gamma$.

Then for every $x$ - $x$ corner $Q$ we can find more than $N / 2$ parallel edges incident to $Q$ since there are $N+1$ edges incident to $Q$. If $N$ is even, then we have a (non-squashed) Scharlemann cycle by Sublemma 5.4. If $N$ is odd, then we have the following two possibilities by Sublemma 5.4.

(i) $\Gamma$ contains a (non-squashed) Scharlemann cycle, or

(ii) the two edges which have the label $x$ at the end points in $Q$ have also the label $x$ at the other end points, and the edges incident to $Q$ are divided into two families of $(N+1) / 2$ parallel edges which connect $Q$ with two corners adjacent to $Q$ on $\partial E$ (see Figure 5.2).

We may assume that all the $x$ - $x$ corners satisfy the condition (ii), because otherwise we can find a non-squashed Scharlemann cycle. Under this assumption we show that every corner on $\partial E$ is an $x-x$ corner.

Let $Q_{0}, Q_{1}, \ldots, Q_{m}$ be the corners on $\partial E$ appearing sequentially in this order. Let $e_{i}$ be the edge on $\partial E$ connecting $Q_{i}$ and $Q_{i+1}$, where $e_{m}$ connects $Q_{m}$ and $Q_{0}$. The edge $e_{i}$ has the tail in the corner $Q_{i}$ and the head in the corner $Q_{i+1}$. We assume that $Q_{0}$ is an $x-x$ corner and show that the next corner $Q_{1}$ is also an $x-x$ corner. The label of the head of $e_{0}$ is $x$ and the label at the tail of $e_{m}$ is also $x$ by the condition (ii). If the tail of $e_{1}$ has the label $x$, then we are done. Assume for a contradiction that the tail of $e_{1}$ does not have the label $x$. In general, suppose that the label at the tail of $e_{i}$ is not $x$ for some $i(1 \leq i \leq m-1)$. Then the head of $e_{i}$ has the label $x$. If the corner $Q_{i+1}$ is an $x-x$ corner, then by the condition (ii), the tail of $e_{i}$ has the label $x$, a contradiction. Hence $Q_{i+1}$ is not an $x-x$ corner. Thus the tail of $e_{i+1}$ does not have the label $x$. Inductively the assumption that the tail of $e_{1}$ does not have the label $x$ implies that the tail of $e_{i}$ does not have the label $x$ for $i=1, \ldots, m-1$. Therefore particularly the head of $e_{m-1}$ has the label $x$, and $Q_{m}$ is an $x-x$ corner (because the tail of $e_{m}$ has the label $x$ ). Hence by the condition (ii), the tail of $e_{m-1}$ has the label $x$, a contradiction. This proves $Q_{1}$ is also an $x$ - $x$ corner, and hence all the corners are $x-x$ corners, all of which satisfy the 


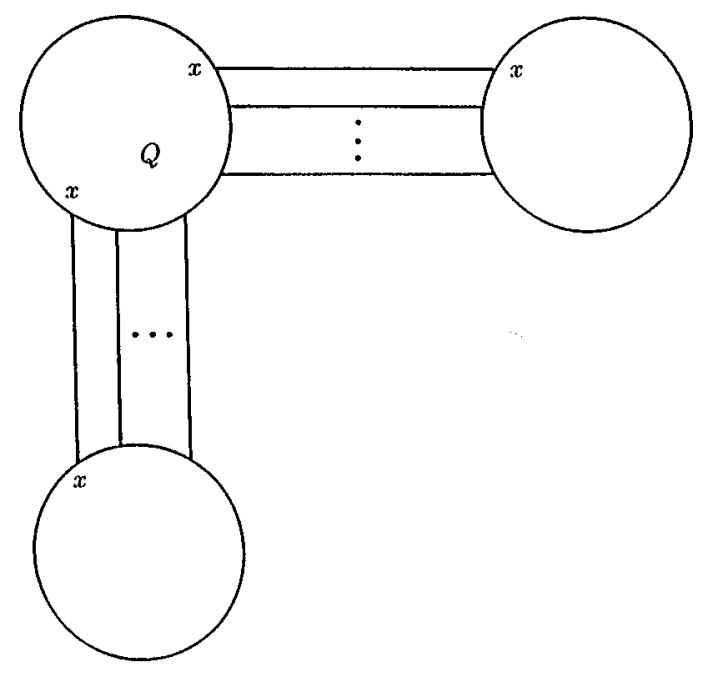

Figure 5.2

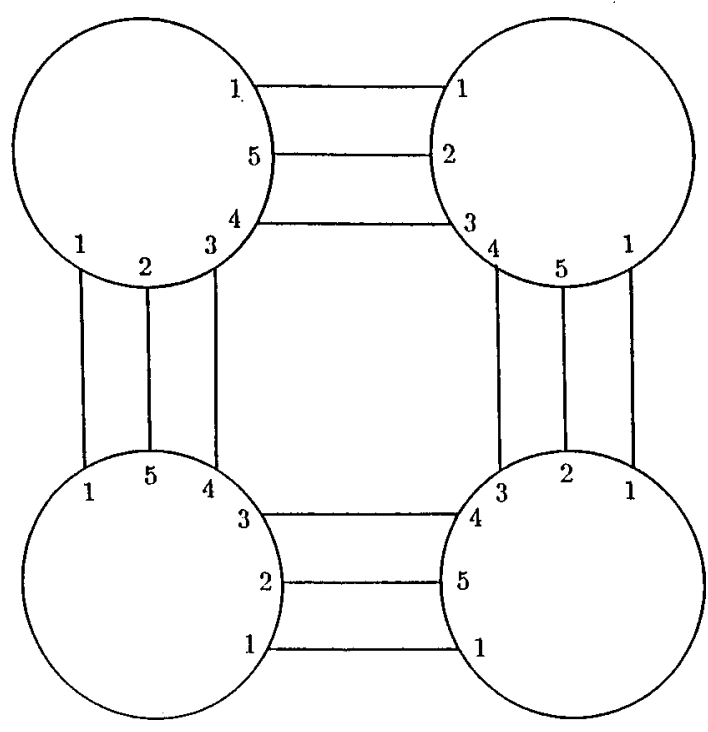

FiguRE 5.3

condition (ii). Then $m$ families of $(N+1) / 2$ parallel edges form $(N+1) / 2 m$-gons (see Figure 5.3).

Hence the innermost $m$-gon is a non-squashed Scharlemann cycle.

Case 2 : There is a diagonal edge $e$ on $\Gamma$, i.e., there is an edge $e$ such that $e$ is incident to an $x$ - $x$ corner $Q$ and has the other end point in a corner $R$ which is not adjacent to $Q$ on the $x$-edge cycle $\sigma$. 


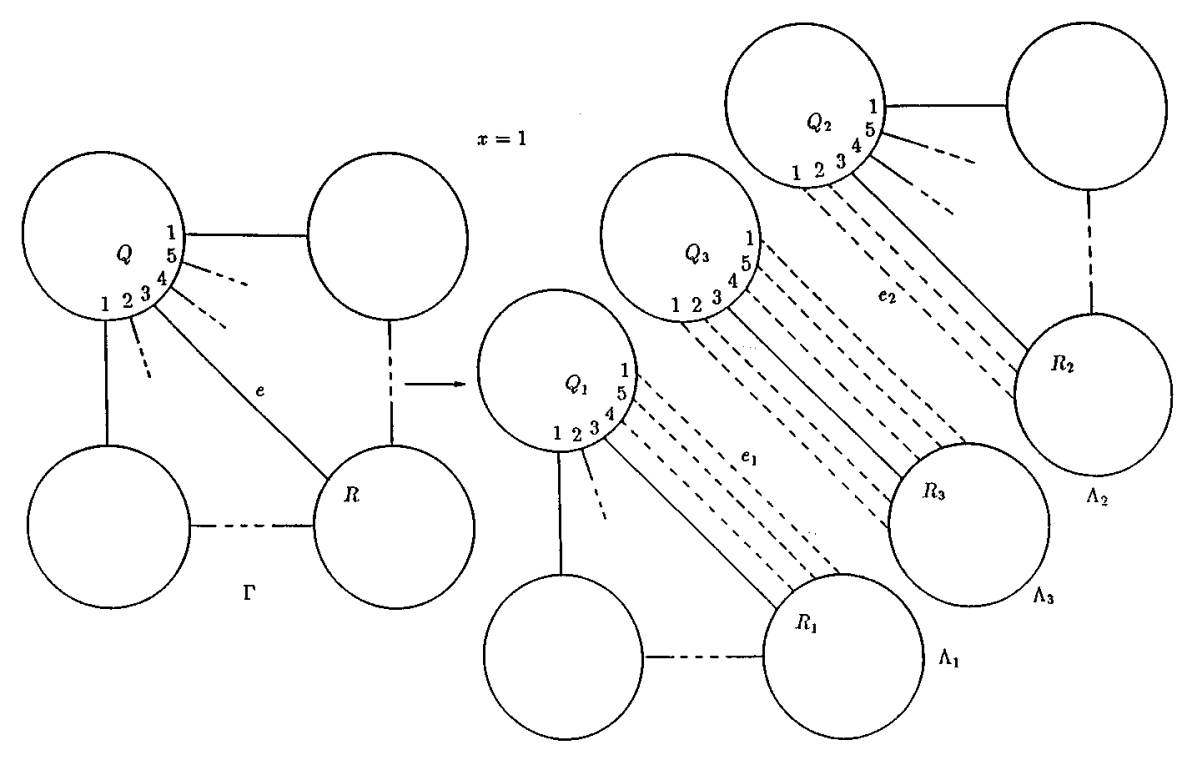

FiguRe 5.4

Let $U$ (resp. $V$ ) be the vertex which contains $Q$ (resp. $R$ ). Let $k$ be the label at the end of $e$ at $Q$. Now we formally construct three graphs. The diagonal edge $e$ divides the disc $E$ into two discs $E_{1}$ and $E_{2}$. Then $\overline{E_{1}} \cap Q$ contains labels $\{x, x+1, \ldots, k\}$ and $\overline{E_{2}} \cap Q$ contains labels $\{k, \ldots, x-1, x\}$. Let $\Lambda_{i}^{\prime}$ be the graph $\left(\Gamma \cap E_{i}\right) \cup \partial E_{i}$ for $i=1,2$. Let $\Lambda_{1}$ (resp. $\Lambda_{2}$ ) be the graph obtained from $\Lambda_{1}^{\prime}$ (resp. $\Lambda_{2}^{\prime}$ ) by adding edges which are parallel to $e$ and incident to $Q$ with labels $k+1, \ldots, x-1, x$ (resp. $x, x+1, \ldots, k-1)$. Let $e_{i} \subset \Lambda_{i}$ be the outermost additional edge. Let $D_{i}$ be the disc bounded by the two edges $e$ and $e_{i}$. We construct the third graph $\Lambda_{3}$ as $\left(\left(\Lambda_{1} \cap D_{1}\right) \cup \partial D_{1}\right) \cup\left(\left(\Lambda_{2} \cap D_{2}\right) \cup \partial D_{2}\right)$, and denote the $\operatorname{disc} \operatorname{int} \overline{D_{1}} \cup \overline{D_{2}}$ by $D_{3}$. The graph $\Lambda_{3}$ consists of two vertices $U, V$ and $N+1$ parallel edges connecting them. See Figure 5.4.

In the graph $\Lambda_{i}$ let $R_{i}$ be the corner of $\partial D_{i}$ on $\partial V$ which contains the end of $e$. We note that $e$ does not have the label $k$ at $R_{i}$ because the weak parity rule holds in $E$. The graph $\Lambda_{i}$ is bounded by an $x$-edge cycle $\sigma_{i}$, and these graphs satisfy the conditions (1)-(4). However these graphs may not satisfy the weak parity rule on $\Lambda_{i}-\left(\sigma_{i} \cup e\right)$. In the graph $\Lambda_{3}$, we can easily observe that

Sublemma 5.5. (1) If the corner $R_{3}$ is also an $x$-x corner, then the graph $\Lambda_{3}$ has just three (possibly squashed) Scharlemann cycles and only one is in $D_{3}$. If $N$ is even, then all the three Scharlemann cycles are squashed. If $N$ is odd, then just one of them (which is in $D_{3}$ ) is non-squashed (see Figure 5.5).

(2) If the corner $R_{3}$ is not an $x-x$ corner, then let $f$ be the edge which has the label $x$ at $R_{3}$. The graph $\Lambda_{3}$ has just one (possibly squashed) Scharlemann cycle between the edges $f$ and $e_{i}$ for $i=1$ and 2, respectively (see Figure 5.5).

Let us assume that $R_{3}$ is also an $x-x$ corner. Recall that the diagonal edge $e$ is not a squashed Scharlemann cycle. Hence by Sublemma 5.5, one of the two graphs $\Lambda_{1}$ and $\Lambda_{2}$, say $\Lambda_{1}$, contains just one squashed Scharlemann cycle $e_{1}$ and contains no more Scharlemann cycles in the $\operatorname{disc} D_{1}$. Then $\operatorname{in} \operatorname{int}\left(\overline{E_{1}} \cup \overline{D_{1}}\right)$, the weak parity 


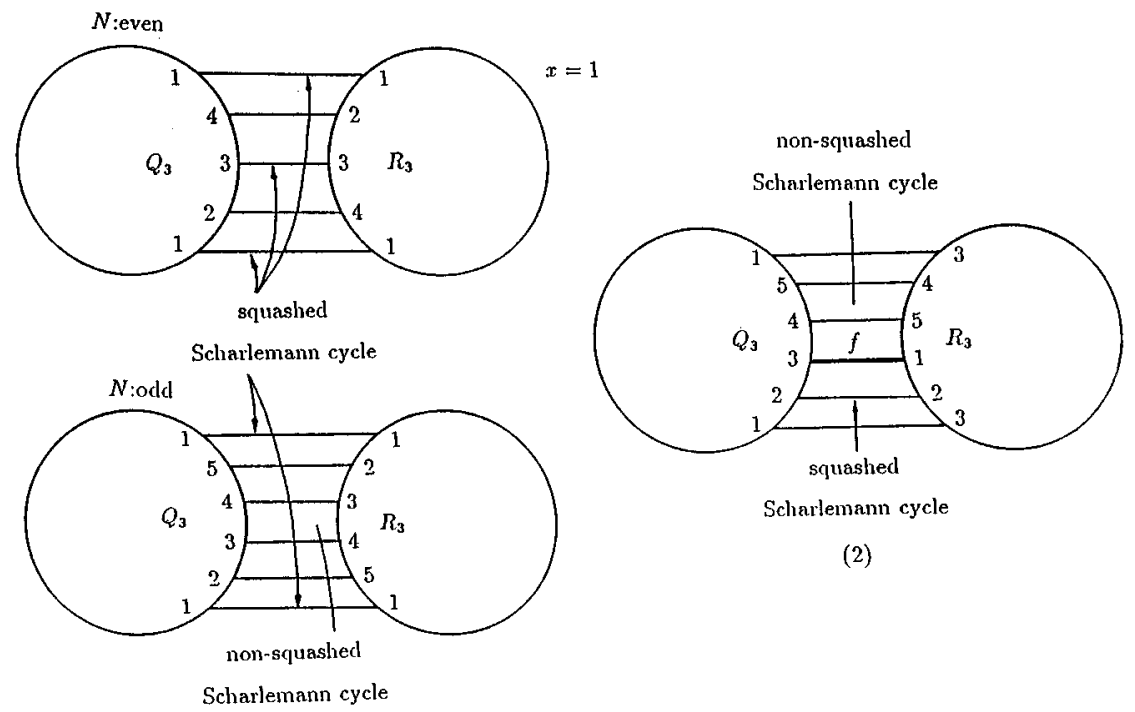

(1)

FiguRE 5.5

rule holds. Hence by the inductive hypothesis, there is a face of a non-squashed Scharlemann cycle in $E_{1} \cup D_{1}$. Since there is no non-squashed Scharlemann cycle in $\overline{D_{1}}$, we can find a face of a non-squashed Scharlemann cycle in $E_{1}$.

Now let us assume that $R_{3}$ is not an $x$ - $x$ corner. Then we divide into two subcases according to the position of $f$ and the diagonal edge $e$ in $\Lambda_{3}$.

First suppose that one of the two graphs $\Lambda_{1}$ and $\Lambda_{2}$, say $\Lambda_{1}$, does not contain a (possibly squashed) Scharlemann cycle in $\overline{D_{1}}$. Then the graph $\Lambda_{1}$ is bounded by the $x$-edge cycle $\sigma_{1}$ and in $\operatorname{int}\left(\overline{E_{1}} \cup \overline{D_{1}}\right)$ the weak parity rule holds. Hence there is a face of a non-squashed Scharlemann cycle in $E_{1}$ by the inductive hypothesis.

Suppose that both graphs $\Lambda_{1}$ and $\Lambda_{2}$ contains just one (possibly squashed) Scharlemann cycle in $\overline{D_{1}}$ and $\overline{D_{2}}$ respectively. Then at least one of the two graphs $\Lambda_{1}$ and $\Lambda_{2}$, say $\Lambda_{1}$, has the edge $f$ in $\overline{D_{1}}$. ( $f$ may be coincide with $e$.) Substituting the edge $f$ for the edge $e_{1}$ in the $x$-edge cycle $\sigma_{1}$, we can obtain a new $x$-edge cycle $\rho$ such that the weak parity rule holds in the face $H$ of $\rho$. By the inductive hypothesis, there is a face of a non-squashed Scharlemann cycle. In any case, since $H \cap D_{1}$ does not contain a face of a Scharlemann cycle, the above one exists in $E_{1}$. This completes the proof of Lemma 5.2.

The proof of Proposition 5.1 is now complete.

\section{Calculation of Euler characteristic - Proof of Theorem 2.1}

The goal in this section is to prove Theorem 2.1.

Lemma 6.1. Suppose that for some label $x$ the graph $G_{i}$ contains more than $\left(n_{i}+\right.$ $\left.t_{i}-\chi\left(F_{i}\left(\gamma_{i}\right)\right)\right)$ sign-preserving edges each of which has the label $x$ at an end point. Then $G_{i}$ contains a non-trivial Scharlemann cycle. 
Proof. Let $\Lambda$ be the subgraph of $G_{i}$ consisting of the above sign-preserving edges and all the vertices of $G_{i}$. The graph $\Lambda$ may have an isolated vertex. Let $f_{d}$ denote the number of disc faces of $\Lambda$. Applying Euler's formula for the graph $\Lambda$ on $F_{i}\left(\gamma_{i}\right)$ we have

$$
n_{i}-\left(n_{i}+t_{i}-\chi\left(F_{i}\left(\gamma_{i}\right)\right)+1\right)+\Sigma \chi(\text { face }) \geq \chi\left(F_{i}\left(\gamma_{i}\right)\right) .
$$

Hence $f_{d} \geq \Sigma \chi($ face $) \geq t_{i}+1$. Thus there is a disc face $D$ of $\Lambda$ such that $\bar{D}$ does not contain a trivial loop of $G_{i}$. In other words there is a great $x$-edge cycle which is not a trivial loop and whose face does not contain a trivial loop of $G_{i}$. This implies that $G_{i}$ contains a non-trivial Scharlemann cycle by Proposition 5.1.

The following is equivalent to Lemma 6.1.

Lemma 6.2. Let $v$ be a vertex of $G_{j}$. Suppose that there are more than $\left(n_{i}+\right.$ $\left.t_{i}-\chi\left(F_{i}\left(\gamma_{i}\right)\right)\right)$ sign-reversing edges incident to $v$. Then $G_{i}$ contains a non-trivial Scharlemann cycle.

The next lemma is a consequence of Lemma 6.1.

Lemma 6.3. Suppose that the graph $G_{i}$ contains more than $\left(n_{i}+t_{i}-\chi\left(F_{i}\left(\gamma_{i}\right)\right)\right) \times$ $n_{j} / 2$ sign-preserving edges. Then $G_{i}$ contains a non-trivial Scharlemann cycle.

Proof. In the graph $G_{i}$ there are $n_{j}$ labels $1, \ldots, n_{j}$, and by the assumption there are more than $\left(n_{i}+t_{i}-\chi\left(F_{i}\left(\gamma_{i}\right)\right)\right) \times n_{j}$ end points of sign-preserving edges. Hence for some label $x$ there are more than $\left(n_{i}+t_{i}-\chi\left(F_{i}\left(\gamma_{i}\right)\right)\right)$ sign-preserving edges each of which has the label $x$ at an end point. Thus Lemma 6.1 implies that $G_{i}$ contains a non-trivial Scharlemann cycle.

Now we can prove Theorem 2.1.

Proof of Theorem 2.1. Suppose that neither $G_{1}$ nor $G_{2}$ contains a non-trivial Scharlemann cycle. In the graph $G_{1}$ there are $\Delta n_{1} n_{2}$ endpoints of the edges around the fat vertices. Lemma 6.2 shows that there are less than or equal to $\left(n_{2}+t_{2}-\chi\left(F_{2}\left(\gamma_{2}\right)\right)\right) n_{1}$ end points of sign-reversing edges in $G_{1}$. Hence there are more than or equal to $\Delta n_{1} n_{2}-\left(n_{2}+t_{2}-\chi\left(F_{2}\left(\gamma_{2}\right)\right)\right) n_{1}-b$ end points of signpreserving edges. By Lemma 6.3 this is less than or equal to $\left(n_{1}+t_{1}-\chi\left(F_{1}\left(\gamma_{1}\right)\right)\right) n_{2}$. Thus we have

$$
\Delta n_{1} n_{2}-\left(n_{2}+t_{2}-\chi\left(F_{2}\left(\gamma_{2}\right)\right)\right) n_{1}-b \leq\left(n_{1}+t_{1}-\chi\left(F_{1}\left(\gamma_{1}\right)\right)\right) n_{2} .
$$

Hence we have the desired inequality :

$$
\Delta \leq 2+\left(t_{1}-\chi\left(F_{1}\left(\gamma_{1}\right)\right)\right) / n_{1}+\left(t_{2}-\chi\left(F_{2}\left(\gamma_{2}\right)\right)\right) / n_{2}+b /\left(n_{1} n_{2}\right) .
$$

\section{REFERENCES}

1. Culler, M., Gordon, C.McA., Luecke, J. and Shalen, P., Dehn surgery on knots, Ann. of Math. 125 (1987), 237-300. MR 88a:57026

2. Goodman-Strauss, C., On composite twisted unknots, Trans. Amer. Math. Soc. 349 (1997), 4429-4463.

3. Gordon, C.McA. and Luecke, J., Knots are determined by their complements, J. Amer. Math. Soc. 2 (1989), 371-415. MR 90a:57006

4. __ Reducible manifolds and Dehn surgery, Topology 35 (1996), 385-409. MR 97b:57013

5. Mathieu, Y., Unknotting, knotting by twists on disks and Property $(P)$ for knots in $S^{3}$, Knots 90 (ed., Kawauchi, A.), Proc. 1990 Osaka Conf. on Knot Theory and Related Topics, de Gruyter (1992), 93-102. MR 93i:57008 
6. Miyazaki, K. and Yasuhara, A., Knots that cannot be obtained from a trivial knot by twisting, preprint (1993).

7. Motegi, K., Primeness of twisted knots, Proc. Amer. Math. Soc. 119 (1993), 979-983. MR 94c:57014

8. Motegi, K. and Shibuya, T., Are knots obtained from a plain pattern always prime ?, Kobe J. Math. 9 (1992), 39-42. MR 93i:57009

9. Rolfsen, D., Knots and Links, Lecture Series 7, Publish or Perish, Berkeley, California, 1976. MR 58:24236

10. Scharlemann, M., Unknotting number one knots are prime, Invent. Math. 82 (1985), 37-55. MR 86m:57010

11. Scharlemann, M. and Thompson, A., Unknotting number, genus, and companion tori, Math. Ann. 280 (1988), 191-205. MR 89d:57008

12. Teragaito, M., Composite knots trivialized by twisting, J. Knot Theory and its Ramifications 1 (1992), 467-470. MR 93k:57023

13. - Twisting operations and composite knots, Proc. Amer. Math. Soc. 123 (1995), 16231629. MR 95f:57024

14. Zhang, X., Unknotting number one knots are prime : a new proof, Proc. Amer. Math. Soc. 113 (1991), 611-612. MR 92a:57017

Department of Mathematical Sciences, University of Tokyo, Hongo 7-3-1, BunkyoKU, TOKYO 113, JAPAN

Department of Mathematics, College of Humanities \& Sciences, Nihon University Sakurajosui 3-25-40, Setagaya-ku, TOKyo 156, Japan

E-mail address: motegi@math.chs.nihon-u.ac.jp 